\title{
QUAND L'EXPERIENTIEL EN LUXE SE CONSTRUIT SUR INSTAGRAM : SYNERGIE OU BATAILLE ENTRE MARQUES ET DIRECTEURS ARTISTIQUES ?
}

\section{Maria MERCANTI-GUERIN}

Maître de conférences HDR, IAE de Paris-Gregor - EA 2474

Christel de LASSUS

Professeur des Universités, IRG, Univ Gustave Eiffel, Université Paris Est Créteil, F-77454

Marne-la-Vallée, France

\section{Christophe BEZES}

Professeur HDR, ISTEC Paris

Montpellier Recherche en Management (MRM), Université de Montpellier c.bezes@istec.fr 


\title{
QUAND L'EXPERIENTIEL EN LUXE SE CONSTRUIT SUR INSTAGRAM : SYNERGIE OU BATAILLE ENTRE MARQUES ET DIRECTEURS ARTISTIQUES ?
}

\begin{abstract}
Résumé
Instagram est devenu le réseau social privilégié des marques de luxe qui y développent leur évènementiel de façon active. Parmi les multiples stratégies de présence sur ce réseau, figure la mise en avant des contenus et des comptes des directeurs artistiques (DA). Or, certains développent des stratégies propres qui rendent floue l'expression des positionnements des marques de luxe pour lesquels ils travaillent.

Une méthodologie innovante, par mobilisation de l'intelligence artificielle et par analyse des contenus et objets postés sur Instagram (textes et « captions ») a permis d'étudier l'expérientiel construit sur Instagram par les DA. Les résultats proposent une typologie de la collaboration entre DA et marques. Ils soulignent la difficulté de gérer de multiples émetteurs sur les réseaux sociaux. Les conclusions de cette recherche ouvrent la voie à une réflexion plus globale sur la difficile gestion des influenceurs dans le secteur du luxe.
\end{abstract}

Mots-clés : luxe, Instagram, expérientiel, directeurs artistiques, IA

\begin{abstract}
Instagram has become the preferred social network for luxury brands that actively develop their events there. Among the many strategies of presence on this network is the highlighting of content and accounts by artistic directors (ADs). However, some develop their own strategies that blur the expression of the positioning of the luxury brands for which they work.

An innovative methodology, by mobilising artificial intelligence and by analysing the contents and objects posted on Instagram (texts and "captions") has made it possible to study the experiential built on Instagram by the AD. The results propose a typology of the collaboration be-tween ADs and brands and underline the difficulty of managing multiple transmitters on social networks. The conclusions of this research pave the way for a more global reflection on the difficult management of influencers in the luxury sector.
\end{abstract}

Key-words: luxury, Instagram, experiential, artistic directors, AI

\section{Introduction}

Sur Instagram, les marques de luxe initient des conversations avec leurs clients via trois canaux : leurs comptes officiels, les influenceurs (blogueurs, vlogeurs, YouTubers...) et les comptes de leurs managers. Ainsi, le directeur artistique ou DA prend-il la parole de façon très scénarisée pour présenter les nouvelles collections, produits, partenariats ${ }^{1}$... Souvent très médiatisé, il contribue à l'expérience de la marque en étant, parfois, plus ou autant connu que cette dernière. Néanmoins, assez peu de recherches académiques en gestion sont consacrées au DA, personnage pourtant central dans cet univers (Hyunjoo, 2015). Ceci est dû à son statut ambigu et aux multiples significations que le terme "Directeur Artistique" peut recouvrir dans d'autres

\footnotetext{
${ }^{1}$ Ipsos, 2019 World Luxury Tracking (WLT), Kolsquare, plateforme pour l'optimisation de campagnes d'Influence Marketing, décembre 2019
} 
domaines que celui de la mode : de planner designer en passant par créateur ou artiste entrepreneur (Pillet-Anderlini, 2016). Alors que les communications Instagram développées par les DA sont en forte progression ${ }^{2}$, il est donc intéressant d'en comprendre l'effet. C'est pourquoi cette recherche propose de mixer un courant de recherche fort en marketing, le secteur du luxe et une personne clé liée à cet univers et source d'un nouvel expérientiel : le DA.

L'apport du DA à la dimension expérientielle du luxe permet de dresser une typologie de la collaboration qu'il entretient avec la marque. Plusieurs questions se posent : est-il un relai efficace des publications de la marque et plus généralement de son univers ? Travaille-t-il plus à promouvoir son image que celle de la marque ? Peut-il parfois être perçu davantage comme un concurrent que comme un allié ? Son positionnement parfois très éloigné de la marque peutil brouiller le positionnement de cette dernière ou au contraire le porter et le moderniser ? L'originalité de notre recherche réside dans la visualisation des relais (ou pas) que le DA peut faire de l'expérience créée en ligne par la marque. La création d'expérience est analysée dans un espace social très concurrentiel pour le luxe (celui d'Instagram) au sein duquel il est possible de quantifier sa capacité à générer de l'engagement ou, au contraire, à publier des contenus à contrecourant de l'univers de la marque. Dans une première partie, nous analysons les liens entre luxe, social et expérientiel. Nous interrogeons les dynamiques d'influence qui traverse ce secteur et les modélisations les plus pertinentes de la "luxemosphère" qui impliquent des approches narratives différentes (Brun et al., 2013) plus adaptées au contexte digital et qui changent la façon de "vivre le luxe". Dans une deuxième partie, une étude empirique couple l'utilisation de l'intelligence artificielle et l'analyse de contenu pour aboutir à une vision globale (images et commentaires) des stratégies sociales des DA et des comptes corporate des marques. Les implications théoriques de cette recherche portent sur une nouvelle méthodologie d'études adaptée à un réseau social visuel et une revisite du concept d'influence expérientielle à l'heure des réseaux sociaux. Les implications managériales vont de la difficile gestion des influenceurs salariés à l'élaboration de stratégies expérientielles congruentes avec l'image de la marque.

\section{Luxe, réseaux sociaux et influenceurs expérientiels}

Les réseaux sociaux permettent au luxe de moderniser son image, de toucher de nouvelles cibles et de faire émerger de jeunes marques. Le contenu publié par les marques a augmenté la perception favorable du secteur, l'image de marque et l'intention d'achat des consommateurs (Kwon et al., 2017). Les effets des stratégies SNSs (social media marketing and brand publishing on social networking sites) portent sur les réactions des consommateurs, la notoriété de la marque et l'attitude, la mise en scène de soi sur les réseaux (Kretz et de Valck, 2010 ; Okonkwo, 2009). Ces expériences sociales renforcent la qualité des relations et l'identification aux marques chez les Millennials (Ladwein et Sanchez, 2018). Plusieurs thématiques émergent

1) la représentation de la marque : publier le contenu de la marque met en avant les innovations et les nouveaux produits, suscite l'appréciation des clients, permet d'intégrer des applications ou d'afficher des contenus associés à la valeur de sa marque plutôt qu'à son domaine d'expertise (Phan et al., 2011).

2) Le shopping social : promotion, catalogue électronique et stories dédiées à la conversion, avant-première exclusive comme promotion des nouvelles collections, campagnes publicitaires en avant-première, diffusion en direct des défilés avec focus sur les produits portés,

\footnotetext{
${ }^{2}$ https://pro.iconosquare.com/resources/instagram-marketing-trends-and-benchmarks-2018
} 
récompenses exclusives pour les fans, jeux-concours, annonces des soldes (Saravanakumar et Suganthalakshmi, 2012).

3) La création de communautés: animation de communautés de marque, accroissement du nombre de fans, détection des insights consommateurs via ces communautés, cocréation et travail sur la viralité, développement de l'engagement (Pentina et Koh, 2012).

4) La captation de la data : mise en place d'outils de social listening adaptés, définition et suivi des KPI, évaluation des campagnes sociales, enrichissement grâce aux réseaux sociaux des CRM on-boarding (Liu et al., 2019).

Parmi ces thématiques, l'expérientiel et l'influence concernent un nombre important de recherches (Tafesse, 2016). L'influenceur peut être source d'expérientiel organisée autour de sa personne ou être médiatisée par cette dernière. Les mécaniques liant évènement fortement expérientiel et influenceur sont couramment pratiquées dans le luxe. Ce dernier a une fonction particulière dans l'entreprise qui lui donne autorité pour être le "chef d'orchestre" de l'expérientiel de marque. C'est cette fonction particulière qui est dévolue au directeur artistique. Dans ce cadre, les liens expérientiel-influenceur sont à étudier.

\subsection{Un luxe de plus en plus expérientiel et social}

Longtemps apanage des élites, les marques de luxe font face à une demande croissante qui les ont conduites à revoir leurs stratégies de conquête. Elles visent une clientèle plus large et, dans les sociétés occidentales, plus tournée vers l'émotion que vers la consommation ostentatoire (De Lassus et Freire 2014 ; Batat, 2019a et b). Dès lors, la compréhension des dimensions de l'expérience est devenue clé sur Internet (Geerts et Veg-Sala, 2014). Le marketing expérientiel engage sensoriellement les consommateurs et ne concerne pas uniquement la propriété (Loussaief et Damay, 2018). L'expérientiel devient de plus en plus social et multidimensionnel. Si les recherches sur l'expérientiel sont très nombreuses (Filser, 2002, Roederer, 2013 ; Pulh et Mencarelli, 2010), le digital et a fortiori Instagram ont fait évoluer le concept (Kwon et al., 2017). Le luxe est l'illustration de cet expérientiel social en plein essor (Batat, 2019b). Le processus de consommation expérientielle ne se limite pas à l'acte d'achat (Holbrook, 2000) mais se déploie avant et après l'interaction avec l'espace marchand (Arnould et al., 2005).

La Fashion Week est, désormais, vécue comme une expérience à $360^{\circ}$ avec applications dédiées, casques virtuels et réalité augmentée qui enrichissent les perceptions des spectateurs sur les réseaux. Les plateformes de réalité virtuelle comme Livit sont utilisées par des marques comme Moschino pour créer des shows sociaux, expérientiels et technologiques. Des drones chez Dolce Gabbana ont ouvert le bal du défilé de la Milan Fashion Week en 2018 pour projeter des images sur grand écran complètement «instagramables ». L'expérientiel social du luxe prend aussi en compte l'interactivité client-marque. Les expériences considérées comme des divertissements (défilés de mode dans les boutiques de créateurs) impliquent souvent un faible degré d'implication de la part du client. Mais l'adjonction d'une ressource permettant des rapports humains interactifs accroît l'implication et l'interactivité. Améliorer l'expérience émotionnelle devient central pour les marques pour générer de l'engagement. Les émotions passent par la fierté des DA qui défilent après le spectacle, la visualisation des couturières au travail, la reconnaissance des mannequins pour le créateur. La modélisation de l'expérientiel permet l'émergence de mécanismes reposant sur la célébrité (Mathys et al., 2016). Particulièrement, le modèle SEM (strategic experientiel modules) couple le social à la création. Ce modèle (Schmitt, 1999) inclut les expériences sensorielles (SENSE), les affectives (FEEL), cognitives créatives (THINK), physiques et comportementales, (ACT), et les expériences d'identité sociale générées par la relation avec un groupe ou une culture de référence 
(RELATE). Toutes ces dimensions se retrouvent dans les modes de communication utilisés dans le luxe.

\subsection{La mise en scène du luxe sur Instagram, vers une perte de contrôle ?}

Pourquoi les clients du luxe se connectent-ils autant sur Instagram qui semble représenter le réseau du luxe et de l'expérience ? Pour Quach et Thaichon, (2017), Instagram apporte des innovations majeures en narration visuelle et de dimensions émotionnelles. Les stories, les carrousels, les publicités dynamiques et les hashtags dédiés aux évènements (fashion week, expositions, inaugurations de boutique ou lancements de produit) ont installé Instagram comme le réseau social de référence des marques de luxe (Tobin et Chulpaiboon, 2016). Par le biais des influenceurs, les marques cherchent à communiquer auprès de leurs clients, notamment sur Instagram, réseau privilégié de l'influence (Abidin, 2016). Les influenceurs sont représentatifs de l'obsession de la mise en scène ciblée et digitale (selfies), de la culture de l'ostentation et de la recherche de l'influence sous toutes ses formes. Les évènements ou les expositions marque (branded exhibitions) facilitent la cocréation de contenus visuels et leur dissémination sur le réseau (Koivisto et Mattila, 2018). Les utilisateurs d'Instagram ont l'engagement et la fidélité à la marque les plus élevés par rapport à Facebook, Twitter, et Snapchat (Phua et al., 2017). La gestion des influenceurs sur Instagram est jugée très souvent problématique. Leur contenu peut échapper aux marques et les instagrammeurs chercher leur intérêt propre (Quach et Thaichon, 2017). La tendance est donc de pratiquer l'employee advocacy (incitations pour les employés de partager les contenus sociaux de la marque) et de disposer d'influenceurs du type employés ambassadeurs (Tsarenko et al., 2018). Le tableau 1 montre l'absence du DA comme objet d'étude. En effet, cet endossement particulier d'une marque par son directeur artistique ne correspond ni à un endossement traditionnel par une célébrité totalement extérieure à la marque, ni à un endossement nouveau par des micro-célébrités (Ambroise et Albert, 2020).

Tableau 1 - Principales recherches traitant des influenceurs du luxe sur Instagram

\begin{tabular}{|l|l|}
\hline \multicolumn{1}{|c|}{ Typologie des influenceurs } & \multicolumn{1}{c|}{ Effets sur les marques sponsorisées } \\
\hline \multicolumn{1}{|c|}{ Micro-célébrités } \\
$\begin{array}{l}\text { Etat d'esprit et ensemble de pratiques d'auto- } \\
\text { présentation dans lesquels les utilisateurs de } \\
\text { manière stratégique vont se mettre en scène afin } \\
\text { d'accroître l'attention (Marwick, 2015). } \\
\begin{array}{l}\text { Individus ordinaires qui atteignent la célébrité } \\
\text { sur Instagram grâce à leur esprit d'entreprise } \\
\text { (Abidin, 2015; Duffy et Hund, 2015). }\end{array}\end{array}$ & $\begin{array}{l}\text { Authenticité, proximité, sympathie. Confiance } \\
\text { via l'identification à l'égard de l'émetteur. } \\
\text { Dégradation possible de l'image de la marque si } \\
\text { les contenus apparaissent trop artisanaux (effets } \\
\text { bricolage ou assemblage). }\end{array}$ \\
\hline \multicolumn{1}{|c|}{ Figures médiatiques, célébrités } \\
$\begin{array}{l}\text { Figures populaires ayant construit leur notoriété } \\
\text { en dehors du réseau Instagram (De Veirman et } \\
\text { al., 2017). Blogueuses de mode, acteurs, } \\
\text { personnalités issues du show-business ou de la } \\
\text { beauté pratiquant l'endossement implicite ou } \\
\text { explicite. (Indvik, 2016; Loureiro et al., 2017) } \\
\begin{array}{l}\text { Les facteurs de succès de ces influenceurs } \\
\text { résident dans la désirabilité sociale, la } \\
\text { bienveillance perçue ressentie par leurs fans, }\end{array}\end{array}$ & $\begin{array}{l}\text { Accroissement de la couverture et de la } \\
\text { notoriété. Bouche à oreille positif. Endossement } \\
\text { et commerce social. Dégradation possible du } \\
\text { caractère exclusif de la marque (influenceurs } \\
\text { multimarques). Perte du sentiment de proximité } \\
\text { (influenceur vu comme un média promotionnel) }\end{array}$ \\
\hline
\end{tabular}




\begin{tabular}{|l|l|}
\hline $\begin{array}{l}\text { leur compétence et leur intégrité (Che et al., } \\
\text { 2017). }\end{array}$ & \\
\hline $\begin{array}{l}\text { Pairs } \\
\text { Influence entre pairs et émergence } \\
\text { d'influenceurs au sein de communautés de } \\
\text { pratique ou de cohortes (Sherman et al., 2016) }\end{array}$ & Imitation ou mimétisme de consommation \\
\hline $\begin{array}{l}\text { Influenceurs virtuels, avatars virtuels } \\
\text { Egéries virtuelles, Brand Avatars - Avatars } \\
\text { imitant les modèles réels ou figurant des } \\
\text { représentations idéales de l'homme. Versions } \\
\text { hybrides et transformées du corps humain } \\
\text { (Andersson et al., 2020) }\end{array}$ & $\begin{array}{l}\text { Modernisation des marques, Retombées Presse, } \\
\text { curiosité. Identification floue par certains } \\
\text { consommateurs du caractère non-humain de } \\
\text { l'influenceur. }\end{array}$ \\
\hline
\end{tabular}

\subsection{Le directeur artistique, un influenceur sous influence?}

Les directeurs artistiques sont aujourd'hui, profondément influencés par les réseaux sociaux dans leurs créations autant que dans la façon de promouvoir leurs collections. Leurs lignes artistiques sont soumises aux règles sociales de réseaux comme Instagram (Rocamora, 2017). Le défilé largement retransmis sur Facebook ou Instagram devient un moment essentiel de la médiatisation de la marque mais aussi du DA. Ce super-influenceur qu'est le DA est chargé de rajeunir des marques traditionnelles, d'intégrer de nouveaux codes visuels, de faire émerger de nouvelles marques. Il devient chef d'orchestre du Live de la marque et de sa présence sur le réseau. Néanmoins, sa marque personnelle forte et son importance au sein de l'entreprise le placent au-dessus d'un certain nombre de sujétions. Son statut le positionne, à la fois, dans et hors de l'entreprise ce qui n'est, pas le cas, des simples influenceurs ou ambassadeurs. Cet ambassadeur employé est un champion de marque dont la gestion s'avère plus délicate qu'un simple ambassadeur influenceur. Certains directeurs artistiques sont-ils trop dissonants ou insuffisamment congruents avec la marque qui les emploie ? L'expérience consommateur qu'ils proposent n'est-elle pas trop éloignée de l'expérientiel habituel de la marque ?

\section{Méthodologie et résultats}

Notre recherche s'appuie sur l'analyse des comptes Instagram des directeurs artistiques (DA) et des marques de luxe pour lesquelles ils travaillent. Le corpus étant particulièrement délicat à définir, les marques de luxe étudiées ont été choisi avec soin : 19 des principales marques de luxe sélectionnées sur Instagram et leurs 20 directeurs artistique (une des maisons ayant deux créateurs). Ont été sélectionnées, de façon exhaustive, toutes les marques de luxe disposant de comptes Instagram de plus de 10.000 membres en 2020 et travaillant avec des directeurs artistiques possédant aussi un compte actif personnel. Deux types d'indicateurs ont été étudiés : 1) les images postées via l'API Cloud Vision de Google ${ }^{3}$. Cet outil d'intelligence artificielle catégorise les éléments qu'il reconnaît dans les images et leur associe des mots les qualifiant (Figure 1). De plus en plus utilisé en recherche (Andrade et al., 2019), il transforme les images en mots et permet de réaliser des analyses de contenus sur des réseaux sociaux très visuels comme Instagram. Les objets détectés dans les images publiées par les DA et leurs marques ont

\footnotetext{
${ }^{3}$ L'API Vision dispose de modèles de machine learning pré-entraînés. Cet outil permet d'attribuer des étiquettes à des images et les classe rapidement dans des millions de catégories prédéfinies. Il peut détecter des objets et des visages, lire du texte imprimé ou manuscrit, et intégrer des métadonnées au catalogue d'images.
} 
été analysés ;2) Les commentaires liés aux publications. Les publications les plus commentées ont été consignées via NCapture du logiciel NVivo, puis leurs textes analysés (captions).

Figure 1 - Design de la recherche via l'API Cloud Vision de Google

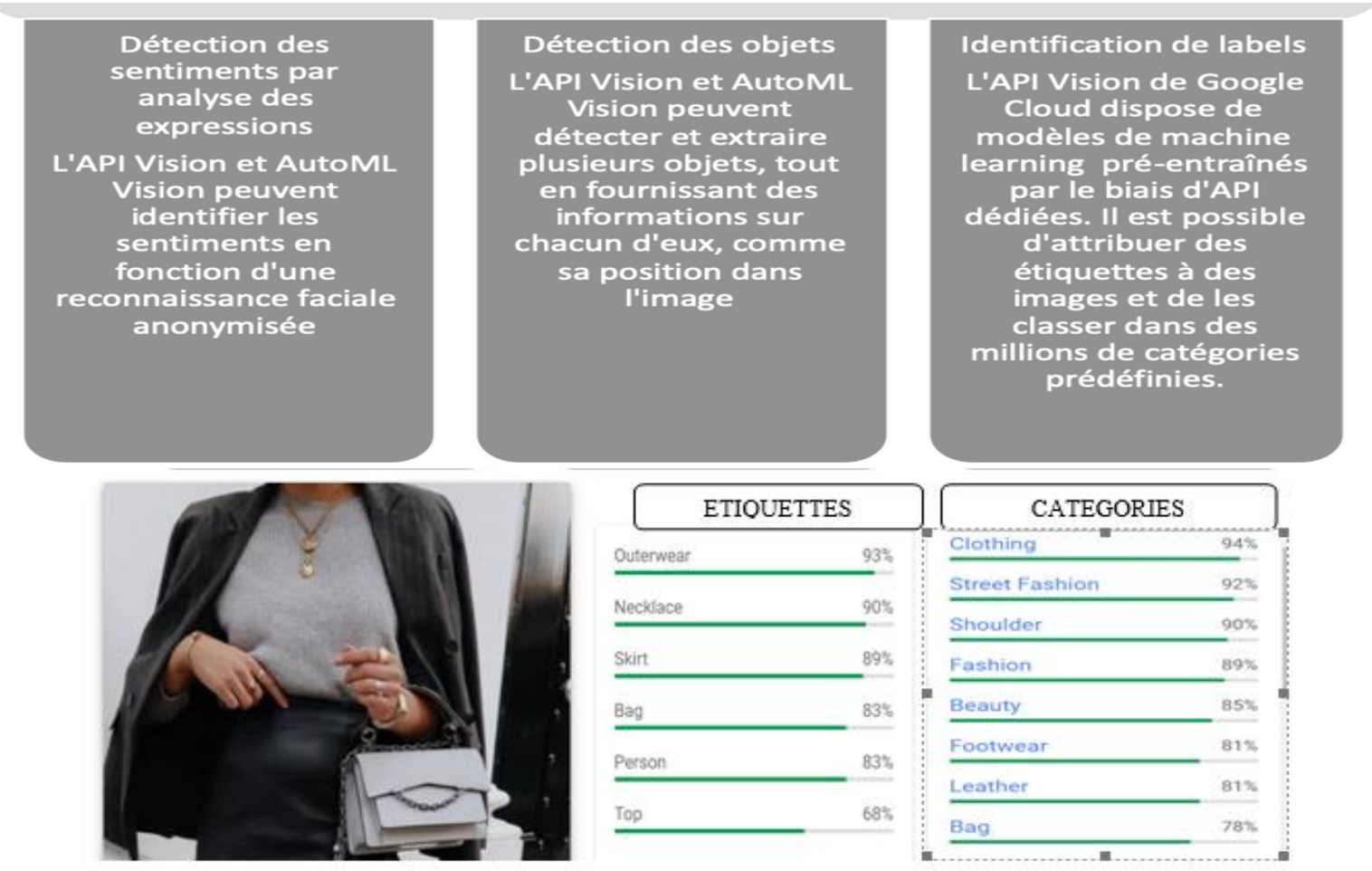

\subsection{Des stratégies de contenu différentes entre directeurs artistiques et marques}

L'analyse de contenu des différentes publications a permis d'établir une classification thématique qui montre une dissociation entre les DA et leurs marques. Quatre types de contenus sont identifiés ${ }^{4}$, certains plus spécifiques des marques, d'autres des DA.

- Amour de la marque, valeurs, nostalgie, sentiments : DA uniquement

- Mode, beauté, modernité, produit : DA et marques en majorité

- Vie privée, quotidien, voyage, recherche d'inspiration dans des univers éloignés de la mode : marques et DA à égalité

- Communication, média, collaborations, communautés, show, collections : marques et DA en majorité

La typologie de contenus des comptes Instagram des DA et des marques du corpus montre une forte représentation des produits (Fashion) et des accessoires (Fashion accessories). De nombreuses publications sont consacrées aux shows et présentations des collections vécus comme des moments incontournables qui rythment l'année. Une part importante des posts célèbre les collaborations avec les influenceuses (célébrités) et leurs couvertures média. Les lieux d'exception chargés de magnifier les produits et de garantir aux shows une bonne couverture médiatique, sont mis en avant (Place to be). Des promotions, présentations de campagnes publicitaires, photos des nouvelles boutiques ouvertes, expérientiel client sont

\footnotetext{
${ }^{4}$ Nous avons effectué des diagrammes de grappes qui permettent de regrouper les sources, les nœuds de thème ou les cas qui partagent des mots, des valeurs d'attribut ou un encodage similaires. Ceci nous a permis de regrouper des thématiques et des individus donc, par exemple un contenu "voyages" avec certains DA ou certaines marques.
} 
intégrées à des posts comportant un lien vers des e-boutiques (Retail). Enfin, la représentation des corps transparaît à la fois dans les comptes de marque et ceux des DA. Ils sont associés à la beauté et source d'inspiration. Le mot "Elégance" revient souvent comme une valeur centrale de l'univers de la mode. Une part non négligeable des publications est consacrée aux valeurs : journée de la femme, philanthropie, lutte contre l'exclusion, affirmation du droit à la différence. La partie Brand Spirit concerne les discours qui réaffirment le statut des marques de luxe. Les termes « Haute Couture, Marque » y sont surreprésentés. La modernité des produits et les mises en avant des produits iconiques inscrivent les marques dans un entre-deux entre tradition et innovation. Les positionnements passent par un sens communautaire développé. On parle de famille Longchamp ou de Balmain Army. Une partie des posts concerne les sources d'inspiration des marques et des AD. Elles puisent dans l'art, le graphisme, l'architecture, la gastronomie, la nature et le travail des matières présenté comme un artisanat de luxe (Matter). Certaines publications ont un aspect nostalgique et privé. Il peut concerner la marque (Longchamp et son histoire) comme les DA. Beaucoup postent des photos de leur enfance et racontent leurs parcours. Ce story telling personnel est enrichi par des vues de leur intérieur, famille ou amis et de passions plus intimes comme la peinture, les voyages, le dessin ou la référence à des univers oniriques. Le privé est également présent dans les posts backstage, les avant-défilés, les équipes (Team Spirit). Enfin, nombre de posts ont trait au métier de DA et au stress généré avant les défilés. Les récompenses et la fierté de travailler pour de grandes marques transparaissent aussi. La communication est très présente chez les DA à travers leurs relations avec les célébrités promouvant leurs marques qu'ils font rentrer dans leur cercle familial et amical (Figure 2).

Figure 2 -Typologie des nœuds identifiés, diagramme de surface ${ }^{5}$

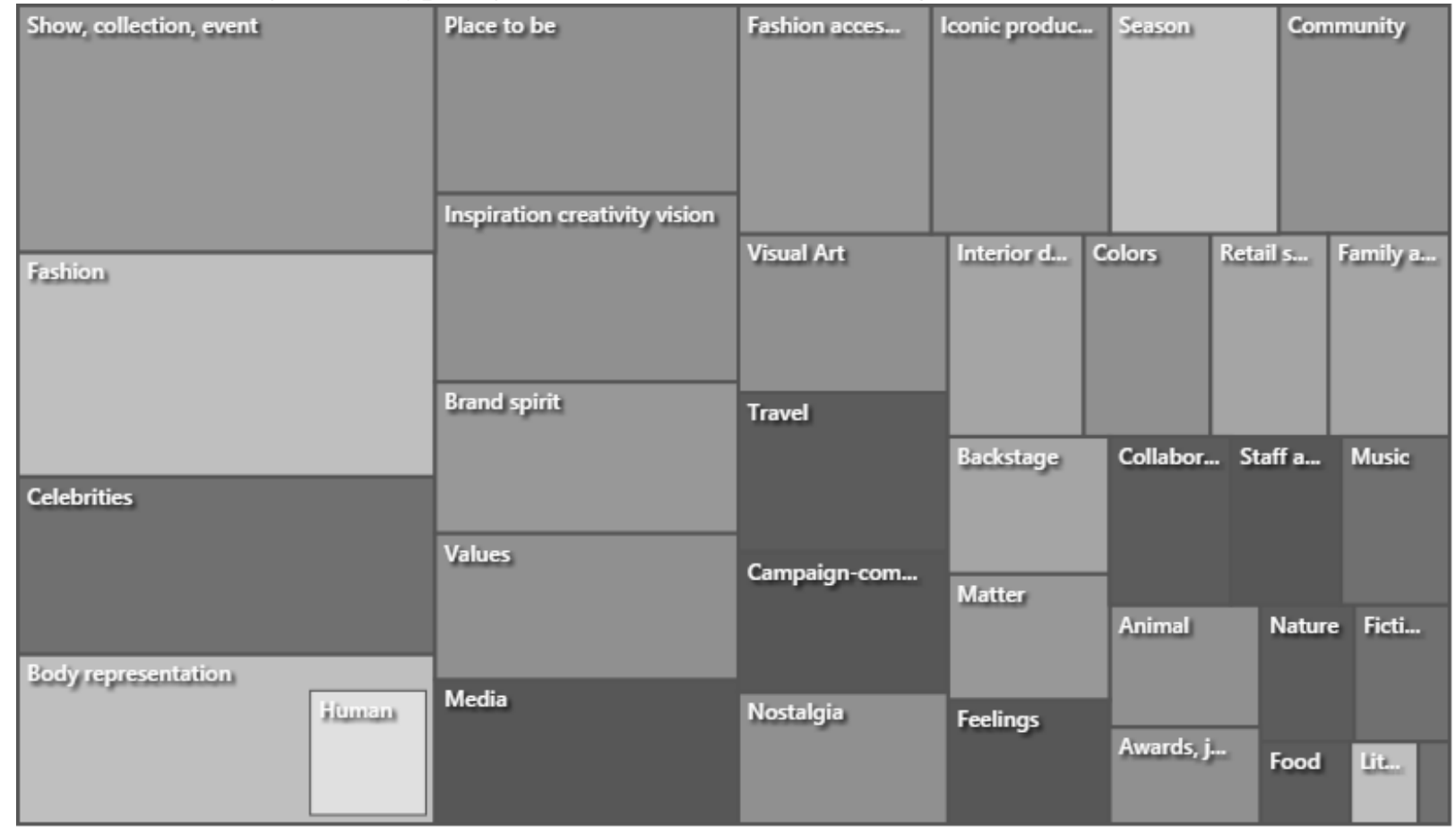

\footnotetext{
${ }^{5}$ Le diagramme de surface permet de comparer des nœuds en fonction du nombre de références qu'ils contiennent.
} Ainsi, la figure 2 montre une place importante du nœud "Show, collection, event" au sein du corpus. 


\subsection{DA/ Marques : des synergies vertueuses et des associations contre-productives}

Des diagrammes de comparaison des thématiques partagées sur les Instagrams des DA et de leurs marques permettent d'établir une classification des collaborations ${ }^{6}$. Elles vont de la synergie vertueuse entre une marque et son DA, à des associations qui peuvent gêner voire brouiller les stratégies sociales des marques. Certains DA mettent en ligne des publications en accord avec la stratégie de contenu de la marque pour laquelle ils travaillent; d'autres exposent des univers très différents, voire disruptifs, par rapport au positionnement de leur marque. Ils brouillent parfois de façon involontaire le positionnement voulu de la marque. Enfin, une petite catégorie de DA s'inscrit en concurrence avec leur marque (détention d'une marque propre, faible proximité avec leur marque employeur...).

\subsubsection{Les DA relais}

Les DA relais (figure 3) partagent de nombreuses thématiques avec leurs marques. Certains peuvent être assimilés à de simples relais des marques; d'autres relaient leur communication en apportant leur univers personnel, ce qui enrichit leur positionnement. Dans les deux cas, ils entretiennent une relation forte avec la marque pour laquelle ils travaillent et sont soumis à un vrai challenge. Cette attitude peut s'expliquer en ce qui concerne Sophie de La Fontaine, descendante du créateur de Longchamp. Un DA comme Pierpaolo Piccioli travaille depuis 1999 pour Valentino. Il a dû prouver sa valeur après le départ chez Dior de Maria Grazia Chiuri. Riccardo Tisci a travaillé pour de nombreuses marques de luxe avant de connaître la consécration chez Valentino. Recruté par Burberry en 2018, il a pour challenge de sauver cette marque. Olivier Rousteing est un DA relais extrêmement actif sur son compte à la fois personnel et professionnel. Réputé comme un maître des réseaux sociaux et de la communication, il procure à Balmain un positionnement avant-gardiste. Le risque de s'appuyer sur les DA Relais pour une marque est qu'une forte congruence peut aboutir à une «valeur positive froide » en termes de construction d'image, c'est-à-dire sans émotion supplémentaire (Mandler 1982, p.22).

\footnotetext{
${ }^{6}$ Deux matériaux ont nourri notre analyse. Google Cloud Vision en traduisant les images partagées sur Instagram en mots a fourni les codes qui sont à la base de l'analyse sémantique. Ces codes pouvaient être la dénomination d'objets, des couleurs ou des sentiments. Ncapture disponible sur le logiciel NVivo a capté et enregistré les différents comptes Instagram étudiés et enrichi notre matériel d'étude. Lors d'une première étape, nous avons effectué un encodage manuel à partir des occurrences les plus fréquentes. Nous l'avons complété par un encodage automatique proposé par NVivo qui utilise des algorithmes d'apprentissage automatique pour effectuer un encodage global des passages de texte contenant des mots similaires à ceux des contenus encodés précédemment. Pour parfaire notre codage, nous avons utilisé les requêtes NVivo pour rechercher et analyser des mots ou des locutions dans nos sources et nœuds. NVivo propose via la recherche textuelle d'afficher toutes les correspondances dans un nœud d'aperçu ce qui nous a permis d'établir les diagrammes de comparaison.
} 
Figure 3 - Le DA relais Allessandro Michele et Gucci (politique de contenu centrée sur le produit, les valeurs de la marque et sa communauté)

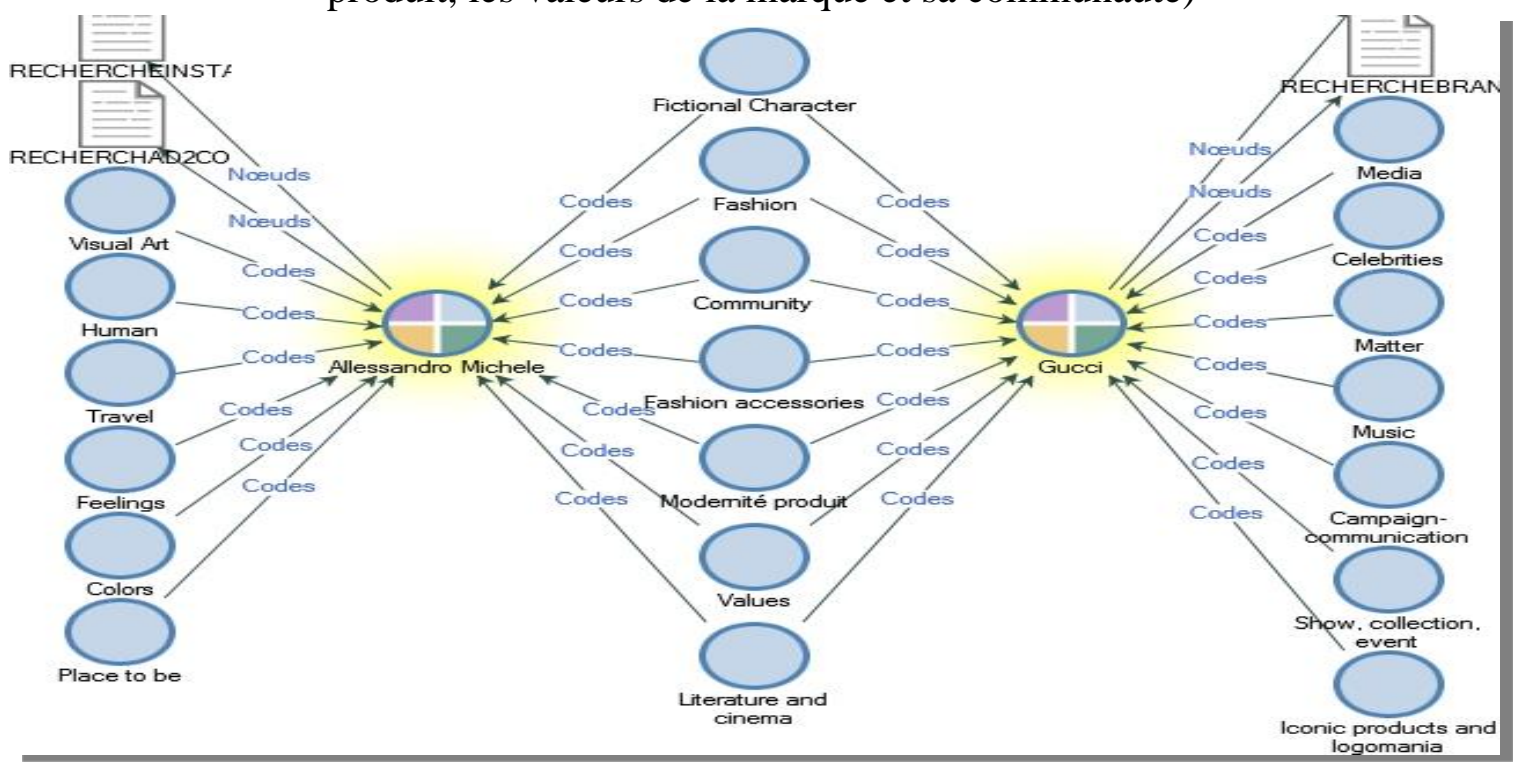

\subsubsection{Les DA Brouillage}

Les DA brouillage partagent peu de contenus avec leurs marques, mais publient par contre, des contenus riches et différenciants sur leurs propres comptes. Ils sont très disruptifs par rapport aux marques qui les emploient et n'hésitent pas à promouvoir leurs propres créations. Leur expérientiel met en scène une vie quotidienne centrée sur l'art, les émotions visuelles et les expériences culinaires. Des associations osées se font entre le métier de la mode et celui d'un créateur à la recherche de sensations dans d'autres univers. Ainsi, passé par de nombreuses maisons avant de rejoindre Vuitton, Nicolas Ghesquière développe-t-il sur Instagram, une personnalité propre influencée par la science-fiction et l'art moderne. Il existe par lui-même avant d'exister pour et par Vuitton. Même si en tant que tels ces DA peuvent apparaître comme crédibles et attractifs, leur discours décalé par rapport à la marque peut être jugé par la cible comme incongruent et affaiblir les éléments centraux de la marque (Kamins et Gupta, 1984).

Figure 4 - Le DA Brouillage Silvia Venturini (faible similarité de contenus avec sa marque et un univers personnel riche et éloigné de son quotidien professionnel)

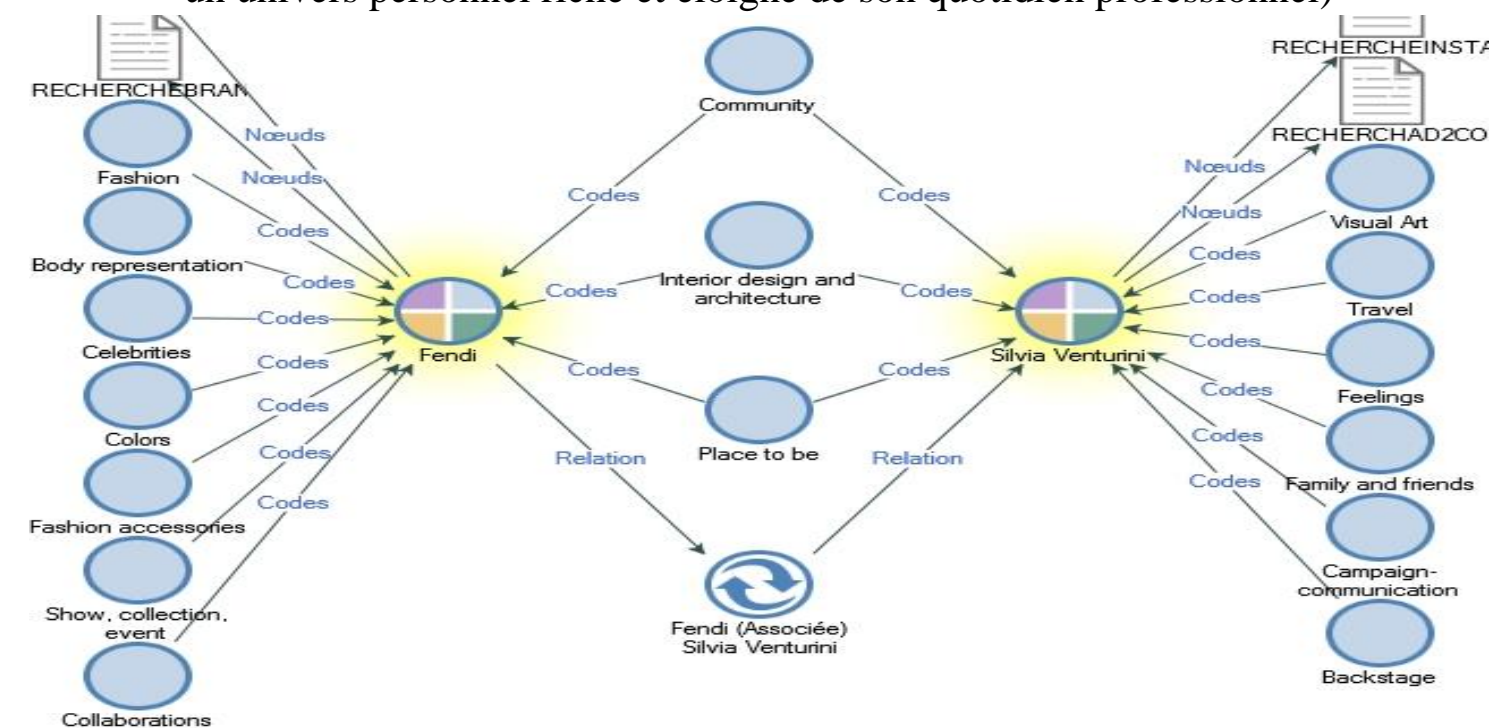




\subsubsection{Les DA Concurrence}

Les DA Concurrence peuvent privilégier sur leurs comptes personnels leurs propres créations. Ils peuvent également partager des univers plus en accord avec des marques pour lesquels ils ne travaillent pas. Paradoxalement, certains DA qui possèdent leurs marques propres servent de sources d'inspiration aux marques sous lesquelles ils sont sous contrat. Les shows organisés reposent, parfois, sur les mêmes sources d'inspiration expérientielles pour la marque employeur et le DA. Le DA Concurrence est, souvent, un influenceur qui se positionne comme une source expérientielle à lui seul. Il est vu comme une star ou un people. Son activité et son discours sur Instagram peuvent être perçus par le public comme une incongruence modérée qui génère un supplément de valeur affective en faveur de leur propre marque. (Bèzes et Mercanti-Guérin, 2017).

Figure 5 - Le DA Concurrence Karl Lagerfeld (grande proximité avec Chanel même si de nombreuses publications poussent sa marque propre)

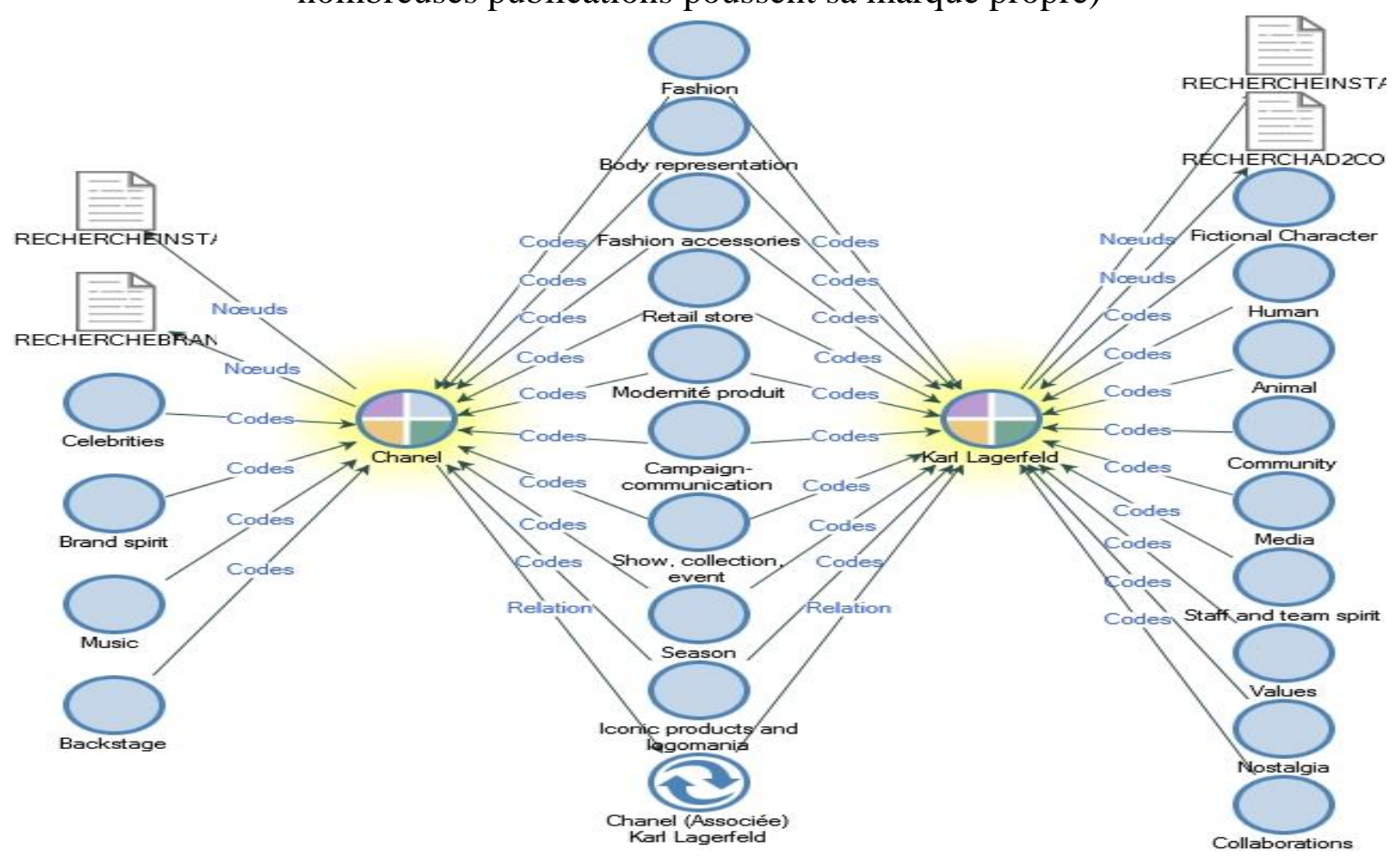

\section{Discussion}

\subsection{Différents types de DA pour différents types d'expérientiels.}

Le tableau 2 permet de cartographier les contenus publiés par les DA en fonction du type d'expérientiel qu'ils proposent à leurs abonnés sur Instagram. Il est issu d'un croisement entre l'analyse de contenu pratiquée et le modèle expérientiel SEM (Schmitt, 1999). Il montre chez les DA un fort expérientiel fondé sur les cinq dimensions du modèle. 
Tableau 2 : Typologie des DA en fonction des types d'expérientiel proposés

\begin{tabular}{|c|c|c|c|c|c|}
\hline \multirow{3}{*}{ 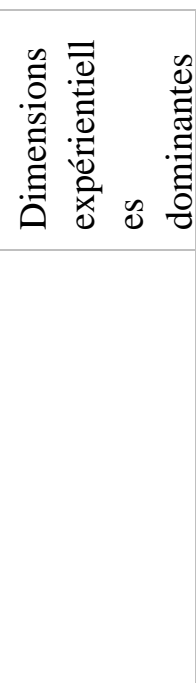 } & SENSE & FEEL & THINK & ACT & RELATE \\
\hline & $\begin{array}{l}\text { Expérience } \\
\text { sensorielle }\end{array}$ & $\begin{array}{l}\text { Expérience } \\
\text { affective }\end{array}$ & $\begin{array}{l}\text { Expérience } \\
\text { cognitive et } \\
\text { créative }\end{array}$ & $\begin{array}{c}\text { Expérience } \\
\text { tirée d'un mode } \\
\text { de vie }\end{array}$ & $\begin{array}{c}\text { Expérience tirée } \\
\text { d'une relation avec } \\
\text { un groupe, une } \\
\text { culture de référence }\end{array}$ \\
\hline & $\begin{array}{l}\text { Mode, } \\
\text { beauté, tissus, } \\
\text { couleurs, } \\
\text { corps, } \\
\text { matières }\end{array}$ & $\begin{array}{l}\text { Amour de } \\
\text { la marque, } \\
\text { valeurs, } \\
\text { nostalgie et } \\
\text { sentiments }\end{array}$ & $\begin{array}{l}\text { Backstage, } \\
\text { séances de } \\
\text { créativité, } \\
\text { mise en scène } \\
\text { des équipes, } \\
\text { visualisation } \\
\text { des ateliers }\end{array}$ & $\begin{array}{l}\text { Voyage, } \\
\text { photographie, } \\
\text { peinture, } \\
\text { recherche } \\
\text { d'inspiration } \\
\text { dans des } \\
\text { univers } \\
\text { éloignés de la } \\
\text { mode }\end{array}$ & $\begin{array}{l}\text { Communication, } \\
\text { média, } \\
\text { collaborations, } \\
\text { communautés, } \\
\text { prises de paroles } \\
\text { publiques, prises de } \\
\text { position sur des } \\
\text { sujets de société }\end{array}$ \\
\hline $\begin{array}{l}\text { Principaux } \\
\text { DA }\end{array}$ & $\begin{array}{l}\text { Waightkeller } \\
\text { Andrew } \\
\text { Michele } \\
\text { Cadwallader }\end{array}$ & $\begin{array}{l}\text { Garcia } \\
\text { Piccioli } \\
\text { Tisci } \\
\text { Kim } \\
\text { Venturini } \\
\text { De La } \\
\text { Fontaine }\end{array}$ & $\begin{array}{l}\text { Vevers } \\
\text { Vacarello } \\
\text { Lagerfeld }\end{array}$ & $\begin{array}{l}\text { Surridge } \\
\text { Jenden } \\
\text { Ramsey-Levi }\end{array}$ & $\begin{array}{l}\text { Gvasalia } \\
\text { Rousteing } \\
\text { Ghesquière }\end{array}$ \\
\hline
\end{tabular}

Concernant l'expérience sensorielle, Casey Cadwallader, DA de Mugler développe avec succès un registre qui met en scène des silhouettes épurées et joue sur des matières fluides enveloppant les corps (latex, laçage, laine). Piccioli (Valentino) travaille sur l'esprit du temps en offrant des expériences nostalgiques et sentimentales (photographies de rue dans les grandes métropoles du monde et sur tous les continents). Vacarello (Yves Saint Laurent) propose des concerts Rock et des spectacles de Paris en plein air où conceptualisation et créativité se rejoignent. Surridge (Cavalli) théâtralise ses espaces d'exposition (Arche de Noé, bestiaire et monde animal). Rousteing (Balmain) développe des rencontres expérientielles en compagnie d'influenceurs et symbolise le DA engagé sur des sujets liés à la responsabilité sociétale de la mode.

\subsection{L'expérientiel : indicateur des jeux de pouvoir et des modes de coordination}

L'organisation particulière des maisons de luxe, à la fois faiseurs de roi et soumis à la volonté d'indépendance de DA qui les surpassent parfois, en capital social pose la question de la coordination des actions entreprises sur les réseaux sociaux. Une lecture par la littérature sur les organisations offre une vision intéressante de ce que beaucoup d'auteurs nomment l'enchevêtrement des modes de coordination. Ces modes sont fondés sur des organisations en réseau et révolutionnés par les réseaux sociaux. Comme le souligne Froehlicher (2001, p. 11), «l'existence de zones d'incertitude dans les champs d'action fournit un degré d'autonomie irréductible à l'acteur par rapport à la structure. Dans cette optique, la frontière de l'organisation est une ressource pour un acteur évoluant à son avantage simultanément dans un milieu interne et externe ». Les acteurs jouent sur une représentation conflictuelle de l'organisation où l'efficacité d'une marque est la résultante d'un équilibre précaire dans les jeux de pouvoir. Dans le cas des marques de luxe, le recrutement de DA prestigieux est souvent salué par les analystes financiers. Une nouvelle génération de DA émerge qualifiée de «producteurs de contenus 
créatifs ». Comme Virgil Abloh ou Kanye West ${ }^{7}$, ces DA peuvent venir d'univers très éloignés de la mode (musique, travail de l'image). Ils arrivent avec une dimension créative, des collaborations et des équipes qui se mettent temporairement au service de la marque. La force de leur réseau est un miroir de leur pouvoir d'influence et non l'inverse. Ainsi, les trois modes de coordination (la hiérarchie, le marché et la coopération) sont remis en question. Les DA n'obéissent à aucune hiérarchie tant que le marché les adoube et valide les bons résultats financiers qu'ils peuvent générer. Dans ce cas, ils sont libres d'imposer leur mode de collaboration et leur type d'expérientiel.

\section{Conclusion}

A l'heure où les influenceurs sont remis en question, cette recherche pointe du doigt les contraintes liées à la gestion de contenu sur Instagram. La multiplication des comptes de marque et associés (fans, salariés, influenceurs) rend problématique la recherche d'une cohérence de positionnement. Le statut particulier d'influenceurs comme les DA rend d'autant plus délicat le rapport de forces qui peut s'instaurer, entre ces influenceurs pratiquant un self branding assumé (Gandini, 2016) et des marques qui fondent leur différence sur leur caractère exclusif.

Les implications managériales de cette recherche mettent en évidence la nécessité de pratiquer une stratégie spécifique en fonction des profils de ces salariés. De nouvelles voies de recherche pourraient être initiées sur le concept d'attachement affectif entre un créateur et sa marque. La prise en compte des récits de vie des DA sur Instagram et de leur histoire personnelle émergent comme des variables essentielles permettant d'expliquer pourquoi et comment une collaboration peut être réellement fructueuse. La typologie proposée de l'influence (relais, brouillage, concurrence) peut être appliquée à d'autres secteurs à l'heure où se multiplient les interrogations sur l'efficacité des stratégies d'influence et la gestion des influenceurs. D'aucuns prédisent même la fin des influenceurs et pourraient être confortés par les résultats de notre étude ${ }^{8}$.

Plus généralement, cette recherche permet d'aller plus loin dans la clarification du concept de champion de marque et, notamment, sa capacité à aider les marques à gérer le changement. Cet effet d'entraînement est illustré par la capacité de certains DA à mettre en avant les équipes (couturiers, casteurs...), à s'inscrire dans l'histoire des marques (clins d'œil à des créations prestigieuses passées) mais aussi à imposer un style plus personnel qui s'intègre ou pas à l'ADN de leur marque employeur.

Enfin, elle offre une illustration de l'utilisation de l'intelligence artificielle appliquée à la recherche sur les réseaux sociaux. Si l'analyse des réseaux sociaux (ARS) s'est, jusque-là, centrée sur les interactions sociales en termes de liens et de nœuds, l'intelligence artificielle permet d'ajouter une dimension plus qualitative de catégorisation des images et de transformation de ces dernières en items. Très utilisée dans la gestion des commentaires, avis, sélection des posts les plus viraux, elle trouve là un nouveau champ d'application et ouvre des voies futures de recherche face à un Internet de plus en plus soumis à la force des images.

En dernier lieu, elle illustre l'indispensable transformation des marques de luxe qui passe par un expérientiel renouvelé. Les DA disruptifs et ré-inspirants pour leurs marques peuvent réussir cette transformation comme ils peuvent échouer. Si le lien entre expérientiel renouvelé et nouveau positionnement de marque est largement observé dans la littérature (Hackley et

\footnotetext{
${ }^{7}$ https://fr.fashionnetwork.com/news/La-nouvelle-generation-des-directeurs-artistiques-ces-createurs-dinfluence, $1081871 . h \mathrm{tml}$

${ }^{8}$ https://nypost.com/2020/06/06/kylie-jenner-and-the-end-of-the-instagram-influencer-business-model/
} 
Tiwsakul, 2006), l'intégration d'émetteurs à la fois salariés et indépendants dans la production d'expérientiels dissonants voire concurrents de la marque n'est pas encore traitée. Il préfigure les problématiques des marques de luxe en termes de gestion de leurs hauts potentiels qui disposent de communautés de fans qui leur sont propres. Il questionne également les recherches portant sur les champions de marque et les mécaniques d'employee advocacy (Morhart, 2009). Fondées sur les seules performances en termes de Reach (couverture sur cible), elles devraient prendre également en compte des indicateurs plus qualitatifs liés aux positionnements des marques et à leurs impératifs de transformation.

\section{Bibliographie}

ABIDIN C. (2016),"Visibility labour: Engaging with Influencers' fashion brands and OOTD advertorial campaigns on Instagram", Media International Australia, n¹61(1), p. 86-100. AMBROISE L. et ALBERT N. (2020), L'endossement par les célébrités : clarifications conceptuelles, synthèse critique et perspectives de recherche, Recherche et Applications en Marketing, Vol. 35, No 2, p. 100-125.

ANDERSSON V. et SOBEK T. (2020), Virtual Avatars, Virtual Influencers \& Authenticity, Master Degree Project.

ANDRADE N. A., RAINATTO G. C., PASCHOAL D. G. E., DA SILVA F. R., et RENOVATO G. (2019), "Computational Vision and Business Intelligence in the Beauty Segment-An Analysis through Instagram", Journal of Marketing Management, $\mathrm{n}^{\circ}$ 7(2), p. 1117.

ARNOULD E. et THOMPSON C. (2005), "Consumer Culture Theory (CCT): twenty years of research", Journal of Consumer Research, Vol. 31, n 1, p. 868-882.

BATAT W. (2019a), The New Luxury Experience, Springer International Publishing.

BATAT W. (2019b), Digital luxury: transforming brands and customer experiences, Sage, London.

BEZES C. ET MERCANTI-GUERIN M. (2017), La similarité en marketing : périmètre, mesure et champs d'application, Recherche et Applications en Marketing, Vol. 32, No 1, p. 86-109.

CHE J. W. S., CHEUNG C. M. K. et THADANI D. R. (2017), "Consumer Purchase Decision in Instagram Stores : The Role of Consumer Trust", Proceedings of the 50th Hawaii

International Conference on System Sciences, p. 24-33.

COTTER K. (2019)," Playing the visibility game: How digital influencers and algorithms negotiate influence on Instagram", New Media \& Society, Vol. 21(4), p. 895-913.

DE LASSUS C. et FREIRE N. (2014), "Access to the luxury brand myth in pop-up stores. A netnographic and semiotic analysis", Journal of Retailing and Consumer Services, Vol. 21, n 1 , p. 61-69.

DE VEIRMAN M., CAUBERGHE V. et HUDDERS L. (2017), "Marketing through instagram influencers: The impact of number of followers and product divergence on brand attitude", International Journal of Advertising, https://doi.org/10.1080/02650487.2017.1348035

DUFFY B.E. et HUND E. (2015), "Having it all" on social media: entrepreneurial femininity and selfbranding among fashion bloggers", Social Media + Society, Vol.1(2), p.1-11.

FILSER M. (2002), " Le marketing de la production d'expérience : statut théorique et implications managériales", Décisions Marketing, Octobre, p.13-22.

FROEHLICHER T. (2001), "La dynamique de l'organisation relationnelle : conventions et réseaux sociaux au regard de l'enchevêtrement des modes de coordination", Finance Contrôle Stratégie.

GANDINI A. (2016), "Digital work: Self-branding and social capital in the freelance knowledge economy", Marketing theory, Vol. 16(1), p. 123-141. 
GEERTS A. et VEG-SALA N. (2014), "Le luxe et Internet : évolutions d'un paradoxe", Management \& Avenir, $\mathrm{n}^{\mathrm{o}}$ 5, p. 111-128.

HACKLEY C. et TIWSAKUL R. (2006), "Entertainment marketing and experiential consumption", Journal of marketing communications, Vol. 12, no 1, p. 63-75.

HOLBROOK M. B. (2000), "The millennial consumer in the texts of our times: Experience and entertainment", Journal of Macromarketing, Vol. 20, nº 2, p. 178-192.

HYUNJOO L. (2015), "Recognizing Design: The Artistic Director as Silent Designer", Design Issues, $\mathrm{n}^{\mathrm{O}} 31(4)$, p. 56-66.

INDVIK L. (2016), The 20 most influential personal style bloggers, Fashionista.

KAMINS M. et GUPTA K. (1984), Congruence between spokesperson and product type: a matchup hypothesis perspective, Psychology \&amp; Marketing, Vol. 11, no 6, p. 569-586.

KOIVISTO E. et MATTILA P. (2018), "Extending the luxury experience to social media-UserGenerated Content co-creation in a branded event", Journal of Business Research.

KRETZ G. et DE VALCK K. (2010), "Pixelize me!': digital storytelling and the creation of archetypal myths through explicit and implicit self-brand association in fashion and luxury blogs", Research in consumer behavior, $\mathrm{n}^{\mathrm{0}} 12$, p. 313-329.

KWON E., RATNESHWAR S. et THORSON E. (2017), "Consumers' Social Media Advocacy Behaviors Regarding Luxury Brands: An Explanatory Framework", Journal of Interactive Advertising, Vol. 17, $\mathrm{n}^{\mathrm{o}}$ 1, p. 13-27.

LADWEIN R. et SANCHEZ A. (2018), "L'ambivalence dans la décision d'acheter des produits de luxe : le cas des millennials", Management \& Avenir, $\mathrm{n}^{\circ}$ 8, p. 155-178.

LIU X., SHIN H., et BURNS A. C. (2019), "Examining the impact of luxury brand's social media marketing on customer engagement: Using big data analytics and natural language processing", Journal of Business Research, May 7.

LOUREIRO S., COSTA I et PANCHAPAKESAN P. (2017), "A passion for fashion: The impact of social influence, vanity and exhibitionism on consumer behavior", International Journal of Retail \& Distribution Management, Vol. 45(5), p. 468-484.

LOUSSAÏ̈EF L. et DAMAY C. (2018), "Le rapport aux vêtements/accessoires de luxe des femmes de 16 à 39 ans dans l'accès vs. la possession exclusive", Management \& Avenir, $\mathrm{n}^{\mathrm{0}} 7$, p. 143-163.

MANDLER G. (1982), The structure of value: accounting for taste, in M.S. Clark et S.T. Fiske (cood), Affect and Cognition: Annual Carnegie Symposium, 17, Hillsdale, Lawrence Erlbaum Associates, p. 3-36.

MARWICK A. E. (2015), "Instafame: Luxury Selfies in the Attention Economy", Public Culture, Vol. 27(1 75), p. 137-160.

MATHY J., BURMESTER A. B. et CLEMENT M. (2016), "What drives the market popularity of celebrities? A longitudinal analysis of consumer interest in film stars", International Journal of Research in Marketing, $\mathrm{n}^{\circ}$ 33(2), p. 428-448.

MORHART F. M., HERZOG W. et TOMCZAK T. (2009), "Rand-specific leadership: Turning employees into brand champions", Journal of Marketing, Vol. 73, n⿳ 5, p. 122-142.

OKONKWO U. (2009),"The luxury brand strategy challenge", Journal of brand management, $\mathrm{n}^{\mathrm{o}} 16(5-6)$, p. 287-289.

PENTINA I. et KOH A.C. (2002), " Exploring social media marketing strategies in SMEs", International Journal of Internet Marketing and Advertising, vol. 7, no 4, p. 292-310.

PHAN M., THOMAS R. et HEINE K. (2011), "Social media and luxury brand management: The case of Burberry", Journal of Global Fashion Marketing, Vol. 2, no 4, p. 213-222.

PHUA J., JIN S. V. et KIM J.J. (2017), "Gratifications of using Facebook, Twitter, Instagram, or Snapchat to follow brands: The moderating effect of social comparison, trust, tie strength, and network homophily on brand identification, brand engagement, brand commitment, and membership intention", Telematics and Informatics", Vol. 34, nº 1, p. 412-424. 
PILLET-ANDERLINI V. (2016), "Création, industrie et communication : la figure du créateur à l'épreuve du storytelling de la création dans la mode", Actes du colloque "De l'injonction à la créativité à sa mise en ouvre », 9-10 avril 2015, Nantes.

PULH M. et MENCARELLI R. (2010), "Muséo-parcs et réenchantement de l'expérience muséale : le cas de la Cité des Arts et des Sciences de Valencia", Décisions marketing, n60, p. 21-31.

QUACH S. et THAICHON P. (2017), "From connoisseur luxury to mass luxury: Value cocreation and co-destruction in the online environment", Journal of Business Research, Vol. 81, p. $163-172$.

ROCAMORA A. (2017), "Mediatization and digital media in the field of fashion", Fashion Theory, Vol. 21, $\mathrm{n}^{\circ} 5$, p. 505-522.

ROEDERER C. (2013), Marketing and experiential consumption, Editions EMS.

SARAVANAKUMAR M. et SUGANTHALAKSHMI T. (2012), "Social media marketing", Life Science Journal, Vol. 9, nº 4, p. 4444-4451.

SCHMITT B. (1999), "Experiential marketing: A new framework for design and communications", Design Management Journal (Former Series), Vol. 10, n 2, p. 10-16.

SHERMAN L. E., RUDIE J. D., PFEIFER J. H., MASTEN C. L., MCNEALY K. et DAPRETTO M. (2014), "Development of the default mode and central executive networks across early adolescence: A longitudinal study", Developmental Cognitive Neuroscience, Vol. $10,148-159$.

TAFESSE W. (2016),"An experiential model of consumer engagement in social media", Journal of Product \& Brand Management, Vol. 25, ${ }^{\circ}$ 5, p.424-434.

TOBIN S. J. et CHULPAIBOON P. (2016), "The role of social connection in satisfaction with Instagram photographs", Translational Issues in Psychological Science, Vol. 2, n 3 , p. 303.

TSARENKO Y., LEO C. et HERMAN H. M. (2018), "When and why do social resources influence employee advocacy? The role of personal investment and perceived recognition", Journal of Business Research, Vol. 82, p. 260-268. 